\title{
NOUVELLE
}

\section{Les antagonistes du récepteur CB1 des cannabinoïdes : une nouvelle approche pour le traitement de la fibrose hépatique}

Fatima Teixeira-Clerc, Boris Julien, Pascale Grenard, Jeanne Tran Van Nhieu, Vanessa Deveaux, Liying Li, Valérie Serriere-Lanneau, Catherine Ledent, Ariane Mallat, Sophie Lotersztajn

Inserm U581, Hôpital Henri Mondor, 51, avenue du Maréchal de Lattre de Tassigny, 94010 Créteil, France.

sophie.lotersztajn@creteil.inserm.fr
> La mortalité par cirrhose représente encore aujourd'hui un problème de santé publique (environ 14000 décès annuels en France à l'heure actuelle). La cirrhose est secondaire à l'accumulation progressive d'une fibrose dans le foie en réponse à une agression chronique, principalement d'origine alcoolique, virale (virus de I'hépatite $B$ ou C), ou métabolique (stéato-hépatite non alcoolique). Réduire ou supprimer l'accumulation de la fibrose représente donc un objectif important de la prise en charge des maladies chroniques du foie. On ne dispose cependant pas, à ce jour, de molécule dont l'effet antifibrosant ait été démontré chez l'homme. Nous avons récemment démontré que l'utilisation de molécules ciblant le système cannabinoïde pourrait représenter une nouvelle approche pour le traitement de la fibrose hépatique.

La fibrose hépatique est un processus de cicatrisation exagérée, qui résulte d'une dérégulation des mécanismes de réparation tissulaire. Elle est la conséquence d'une production excessive de matrice extracellulaire et d'une faillite des voies de dégradation des composants matriciels. Les cellules étoilées du foie et les fibroblastes portaux jouent un rôle majeur dans le processus de fibrogenèse. Au cours des hépatopathies chroniques, ces cellules acquièrent un phénotype myofibroblastique sous l'action de cytokines et de facteurs de croissance produits dans leur environnement. Ces myofibroblastes prolifèrent et s'accumulent dans le foie, notamment dans les zones de nécrose, et synthétisent des cytokines, des chimiokines, des facteurs de croissance ainsi que les composants de la matrice extracellulaire et les molécules bloquant leur dégradation

\section{0 à 20 ans}

\section{Foie normal}

Agression chronique

(virale, alcoolique, métabolique)

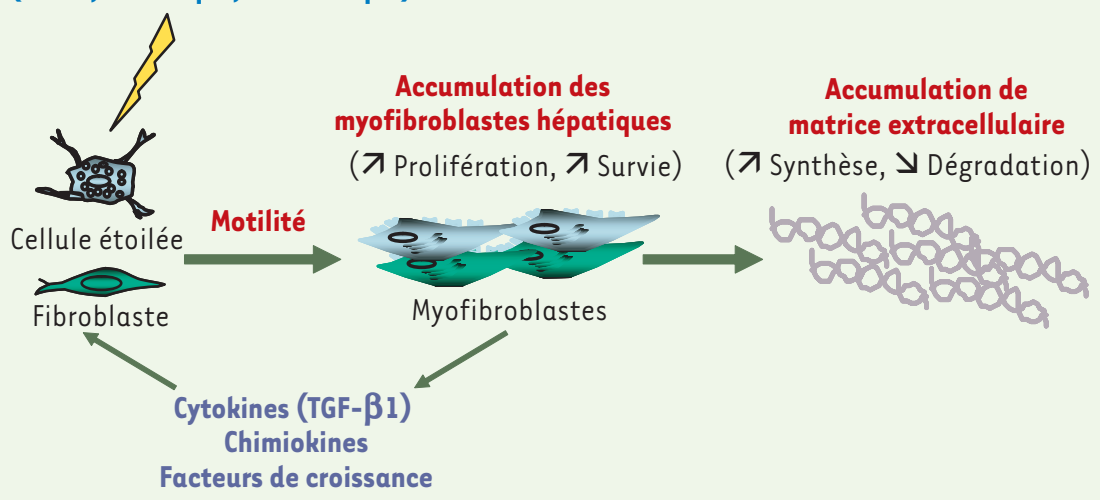

Figure 1. Propriétés fibrogéniques des myofibroblastes. La cirrhose est la conséquence de l'accumulation progressive d'une fibrose dans le foie, souvent sur 10 à 20 ans, en réponse à une atteinte chronique, principalement d'origine alcoolique, virale ou métabolique. Au cours des maladies chroniques du foie, les cellules étoilées du foie et les fibroblastes portaux acquièrent un phénotype myofibroblastique sous l'action de cytokines, de chimiokines et de facteurs de croissance. Ces myofibroblastes migrent vers les zones lésées, prolifèrent et synthétisent les constituants de la matrice extracellulaire, ainsi que les inhibiteurs de leur dégradation. 
(Figure 1) [1]. Parmi les facteurs favorisant l'accumulation de matrice extracellulaire, le TGF- $\beta 1$ (transforming growth factor- $\beta 1$ ) est considéré comme le principal médiateur profibrogénique. Les myofibroblastes hépatiques, en raison de leur rôle majeur dans la fibrogenèse hépatique, représentent une cible privilégiée des molécules antifibrosantes. À cet égard, les principales stratégies antifibrosantes visent à réduire l'accumulation des cellules fibrogéniques en bloquant leur prolifération ou en déclenchant leur apoptose, et/ou à diminuer l'accumulation de la fibrose, en bloquant la synthèse de composants matriciels ou en favorisant leur dégradation [1].

Les cannabinoïdes présents dans la marijuana agissent par l'intermédiaire de deux récepteurs, CB1 et CB2, qui sont également activés par des molécules endogènes, les endocannabinoïdes. Les récepteurs CBl, majoritairement exprimés dans le cerveau, relaient les effets psychoactifs du cannabis, mais exercent de nombreux autres effets centraux et périphériques. Les récepteurs CB2, prédominant dans les cellules du système immunitaire, sont responsables des effets immunorégulateurs, le plus souvent anti-inflammatoires des cannabi- noïdes $[2,3]$. L'identification récente des récepteurs $C B 1$ et $C B 2$ dans un grand nombre de tissus et la mise en évidence de nouvelles fonctions de ces récepteurs ouvrent cependant des voies thérapeutiques nouvelles. Ainsi, le rimonabant, antagoniste sélectif du récepteur CBI vient d'obtenir un avis favorable de l'Agence européenne du médicament dans le traitement du surpoids et des facteurs de risque cardio-métaboliques associés $[4,5]$. Par ailleurs, des études précliniques montrent l'efficacité des agonistes du récepteur CB2 dans le traitement de l'athérosclérose et de l'ostéoporose $[6,7](\rightarrow)$.

Nous avons observé que l'expression des récepteurs $C B 1$ et $C B 2$ est fortement induite dans les zones de fibrose au cours de la cirrhose chez I'homme, notamment dans les myofibroblastes hépatiques $[8,9]$, alors qu'ils sont peu exprimés dans le foie humain normal. Nous avons mis en évidence les propriétés anti-fibrogéniques du récepteur CB2, par un mécanisme impliquant l'inhibition de la prolifération et l'induction de l'apoptose des myofibroblastes hépatiques [8]. Cependant, nous avions également rapporté que l'usage quotidien de cannabis accélère l'évolu- tion vers la cirrhose chez les patients atteints d'hépatite chronique C [10]. Ces résultats nous ont conduit à évaluer le rôle du récepteur CBI dans la progression de la fibrose, en étudiant les conséquences de son invalidation génétique et pharmacologique dans trois modèles expérimentaux de physiopathogénie différente, l'induction d'une hépatite toxique par administration chronique de tétrachlorure de carbone ou de thioacétamide, et la cholestase par ligature de la voie biliaire principale [9]. Dans les trois modèles, nous avons démontré que l'administration per os de rimonabant prévient la fibrogenèse, en réduisant l'accumulation des myofibroblastes hépatiques et la production de TGF- $\beta 1$ hépatique. L'ensemble de ces résultats a été confirmé dans les trois modèles, à l'aide de souris déficientes en récepteur $C B 1$, qui présentent une fibrose réduite par rapport aux souris sauvages. L'étude des mécanismes impliqués dans les effets antifibrogéniques du rimonabant a révélé que la molécule diminue l'accumulation des myofibroblastes hépatiques en inhibant leur prolifération, à la fois in vivo et dans des myofibroblastes hépatiques en culture. Ces effets antiprolifératifs du rimonabant reposent sur l'inhibition de la PI3K/Akt et ERK,

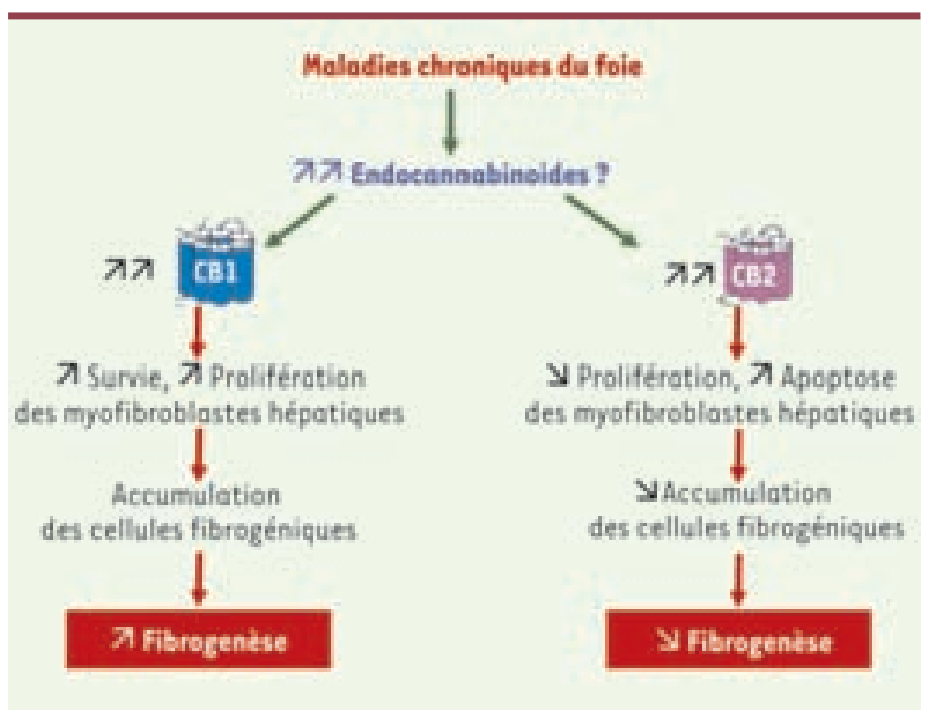

Figure 2. Régulation de la fibrogenèse hépatique par le système endocannabinoïde. Au cours des maladies chroniques du foie, l'élévation de la production d'endocannabinoïdes (dont la nature reste à déterminer) est associée à une augmentation de l'expression des récepteurs $C B 1$ et $C B 2$. Les récepteurs $C B 1$ et $C B 2$ exercent des effets opposés sur la fibrogenèse : le récepteur $C B 1$ favorise la fibrogenèse en augmentant l'accumulation des myofibroblastes hépatiques; à l'inverse, le récepteur CB2 est antifibrogénique en inhibant l'accumulation de ces cellules. Ces résultats suggèrent que l'utilisation combinée d'antagonistes du récepteur CB1 et d'agonistes du récepteur CB2 pourrait constituer une nouvelle approche dans le traitement de la fibrose hépatique. 
deux voies de signalisation contrôlant la prolifération et la survie cellulaire. Ces résultats mettent en évidence les propriétés profibrogéniques du récepteur CBl. Ils démontrent également que I'utilisation d'antagonistes du récepteur CBI tels que le rimonabant pourrait offrir de nouvelles perspectives dans le traitement de la fibrose hépatique au cours des maladies chroniques du foie. Cette étude, associée à nos travaux antérieurs démontrant les effets antifibrosants des récepteurs CB2 [8], suggère qu'une approche antifibrosante optimale pourrait combiner l'utilisation d'antagonistes du récepteur $C B 1$ et d'agonistes du récepteur CB2 (Figure 2). $\diamond$
CBI cannabinoid receptor antagonists: a novel approach for the treatment of liver fibrosis

\section{RÉFÉRENCES}

1. Lotersztajn S, Julien B, Teixeira-Clerc F, et al. Hepatic fibrosis: molecular mechanisms and drug targets. Annu Rev Pharmacol Toxicol 2005 ; 45 : 605-28.

2. Mallat $A$, Lotersztajn $S$. Endocannabinoids as novel mediators of liver diseases. J Endocrinol Invest 2006; $29: 58-65$

3. Di Marzo V, Bifulco M, De Petrocellis L. The endocannabinoid system and its therapeutic exploitation. Nat Rev Drug Discov 2004 ; 3 : 771-84.

4. Van Gaal LF, Rissanen AM, Scheen AJ, et al. Effects of the cannabinoid-1 receptor blocker rimonabant on weight reduction and cardiovascular risk factors in overweight patients : 1-year experience from the RI0Europe study. Lancet 2005 ; 365 : 1389-97.

\section{NOUVELLE}

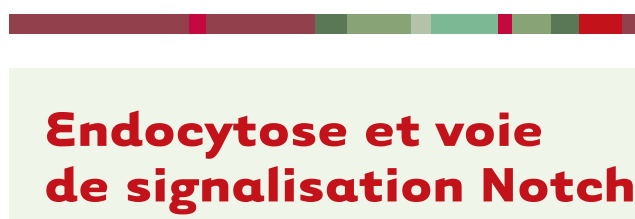

Christel Brou, Frédérique Logeat

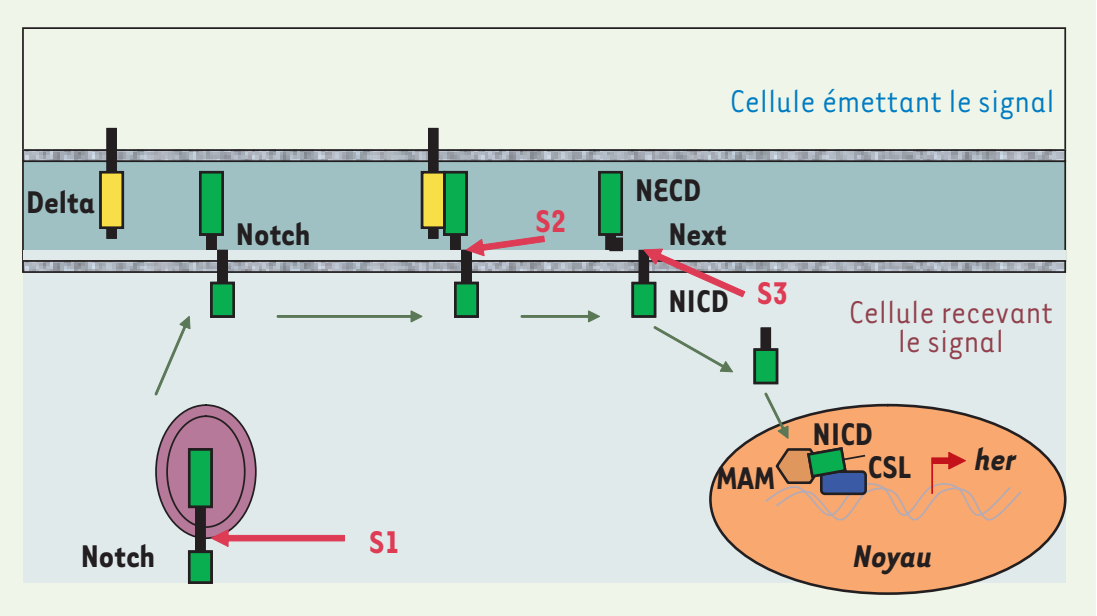

Figure 1. Schéma simplifié de la voie de signalisation Notch. Pendant la maturation de la protéine, Notch subit un premier clivage par la furine (S1). Après l'interaction avec son ligand, ici Delta, Notch subit un second clivage (S2) par une métalloprotéase TACE. Le domaine extracellulaire (NECD) du récepteur Notch est relargué, et le fragment tronqué du domaine extracellulaire restant devient le substrat du complexe $\gamma$-sécrétase qui libère le fragment intracellulaire du récepteur (NICD). Celui-ci migre dans le noyau où il forme un complexe activateur de la transcription avec CSL et MAM (mastermind) qui active les gènes cible de la famille hes/hey (hairy enhancer of split) (d'après [13]).
5. Despres JP, Golay A, Sjostrom L. Effects of rimonabant on metabolic risk factors in overweight patients with dyslipidemia. N EnglJ Med 2005 ; 353 : 2121-34.

6. Steffens S, Veillard NR, Arnaud C, et al. Low dose oral cannabinoid therapy reduces progression of atherosclerosis in mice. Nature 2005 ; 434 : 782-6.

7. Ofek 0 , Karsak M, Leclerc $N$, et al. Peripheral cannabinoid receptor, $\mathrm{CB} 2$, regulates bone mass. Proc Natl Acad Sci USA 2006 ; 103: 696-701.

8. Julien B, Grenard P, Teixeira-Clerc F, et al. Antifibrogenic role of the cannabinoid receptor $C B 2$ in the liver. Gastroenterology 2005; $128: 742-55$

9. Teixeira-Clerc F, Julien B, Grenard P, et al. CB1 cannabinoid receptor antagonism : a new strategy for the treatment of liver fibrosis. Nat Med 2006; $12: 671-6$.

10. Hezode C, Roudot-Thoraval F, Nguyen S, et al. Daily cannabis smoking as a risk factor for progression of fibrosis in chronic hepatitis C. Hepatology 2005 ; 42 : 63-71
Unité de Signalisation Moléculaire et Activation Cellulaire, URA 2582, CNRS, Institut Pasteur, 25, rue du Docteur Roux, 75724 Paris Cedex 15, France.

\section{cbrou@pasteur.fr}

> Depuis C. elegans jusqu'aux vertébrés supérieurs, la voie de signalisation Notch contrôle la compétence de multiples types cellulaires à se différencier. L'activation du récepteur transmembranaire Notch (un gène chez la drosophile, 4 chez les mammifères) est déclenchée par son interaction avec un ligand transmembranaire de la famille DSL (Delta ou Serrate chez la drosophile, Delta 1, 3, 4, ou Jagged 1, 2 chez les mammifères) présenté par une cellule voisine. Le récepteur subit alors deux coupures protéolytiques successives, dans son domaine extracellulaire par une protéase de la famille ADAM (a disintegrin and metalloprotease), puis au sein du domaine transmembranaire par un complexe multiprotéique, la $\gamma$-sécrétase (Figure 1). Le domaine intracellulaire du récepteur est ainsi libéré dans le cytoplasme et migre dans le noyau, où il interagit avec la protéine CSL (CBFl, aussi appelé RBP-Jא chez 
les vertébrés, $\mathrm{Su}(\mathrm{H})$ chez la drosophile, et Lag-1 chez C. elegans) pour reconstituer un activateur transcriptionnel. Les ligands comme les récepteurs sont soumis à des processus comparables de clivages protéolytiques successifs et d'endocytose, sans que, concernant les ligands, leurs rôles soient encore bien compris. L'importance de l'endocytose des ligands et du récepteur Notch pour la signalisation a été initialement décrite chez la drosophile [1] grâce à l'utilisation de mutants pour la dynamine, et fait l'objet de nombreux travaux récents.

\section{Endocytose du récepteur Notch}

L'endocytose du récepteur Notch est nécessaire pour le maintien d'une quantité fixe de récepteurs activables à la membrane, en ciblant et accompagnant les molécules surnuméraires ou défectueuses vers la dégradation, mais elle intervient aussi après activation du
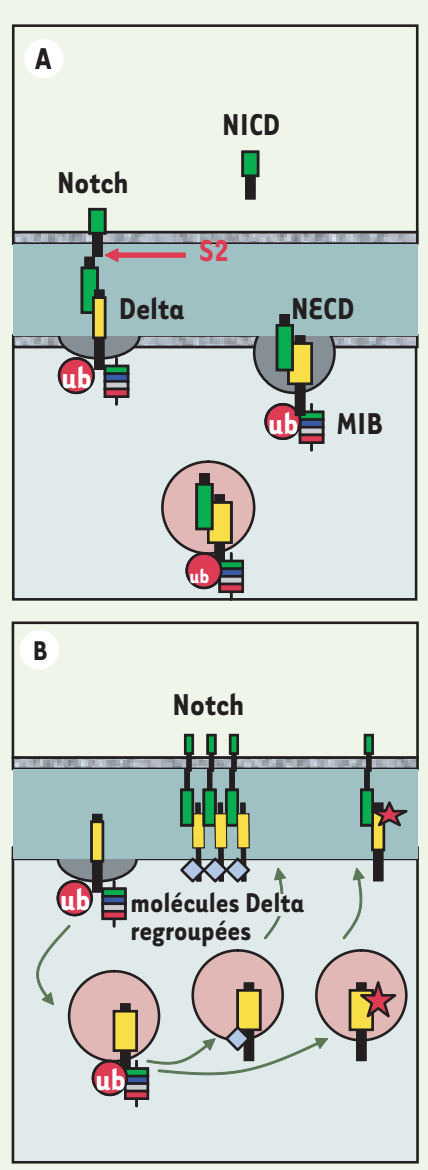

récepteur pour le diriger vers le compartiment où peut agir la $\gamma$-sécrétase. Nous avons montré que le récepteur Notch, après activation, doit être internalisé et mono-ubiquitinylé pour être clivé par la $\gamma$-sécrétase [2]. Chez la drosophile, Sanpodo, une molécule à 4 domaines transmembranaires, joue probablement un rôle positif dans l'activation de Notch en régulant son internalisation de façon antagoniste à Numb [3].

Lorsqu'il n'est pas activé, le récepteur Notch est internalisé et transporté dans des vésicules d'endocytose jusqu'aux lysosomes. Une des premières étapes de ce voyage est l'ubiquitinylation de Notch. Ce signal encore mal caractérisé lui permet probablement d'être trié par interaction avec Hrs/Vps27, puis dirigé vers les vésicules contenant les complexes ESCRTI (endosomal sorting complex required for transport) puis ESCRT-II et ESCRT-III. Les protéines sont alors invaginées dans des petites vésicules au sein des MVB (corps multivésiculaires) et finalement d é g r a dées après fusion des MVB avec les lysosomes. Chez la drosophile, des mutants pour plusieurs des molécules impliquées dans ces trafics bloquent effectivement la dégradation du récepteur, ce qui explique qu'ils provoquent une augmen- tation de l'activité Notch. C'est le cas pour Vps25, Vps22, et Vps32 [4, 5]. En revanche, si les voies d'endocytose sont bloquées plus en amont, par diminution de Hrs, Avl (homologue des syntaxines 7 ou 12, localisé dans les endosomes précoces) ou Rab5, Notch s'accumule à la surface cellulaire mais son activité globale n'augmente pas [6]. Ces résultats sont compatibles avec l'idée selon laquelle le récepteur, qu'il soit activé par liaison du ligand ou pas, subit le processus d'endocytose par une voie dépendant de Rab5 et Avl, et qu'une étape de tri a alors lieu. Selon l'état d'ubiquitinylation du récepteur ou son association avec certaines molécules, il pourrait alors poursuivre sa route vers l'activation ou la dégradation. L'étude des ubiquitinylations subies par le récepteur, et des $\varepsilon 3$ ubiquitine ligases spécifiques de chacune d'entre elles permettra d'élucider ces phénomènes. L'ubiquitinylation par Nedd4 ou Itch/Su(Dx) et cbl pourrait permettre au récepteur d'être dégradé, tandis que son unique mono-ubiquitinylation lui serait nécessaire pour être reconnu par le complexe $\gamma$-sécrétase et donc activer ses gènes cibles.

\section{Endocytose des ligands}

Des études génétiques menées chez la drosophile et le poisson zèbre ont montré qu'un certain nombre de gènes régulaient l'activité des ligands [7]. Ils agissent sur l'endocytose des ligands et sont indispensables à l'activation du récepteur porté par une cellule voisine. Neuralized et Mind bomb, deux $\varepsilon 3$ ubiquitine ligases, sont conservées chez les mammifères, et ont des fonctions partiellement redondantes qui permettent le recrutement des ligands et le transfert de l'ubiquitine sur

\section{Figure 2. Différents modèles proposés pour le rôle de l'endocytose de Delta dans le fonctionnement} de la signalisation Notch. A. L'endocytose de Delta facilite le second clivage (S2) du récepteur Notch. B-D. L'endocytose de Delta et son recyclage résulte en une présentation des molécules Delta plus efficace. B. Le recyclage de Delta entraîne son regroupement avec d'autres molécules ainsi qu'avec des cofacteurs et sa localisation dans un microdomaine, ou sa modification (étoile rouge) dans le domaine extracellulaire facilitant son interaction avec le récepteur Notch. $C$. Après son endocytose, les ligands Delta sont internalisés dans des corps mutivésiculaires (MVB), et via des exosomes, relarguent plusieurs molécules Delta groupées (d'après [13]). 